\title{
EFFECTIVENESS OF VELSCOPE AND VIZILITE PLUS SYSTEMS IN DIAGNOSTICS OF ORAL LESIONS
}

\author{
N. Nikolov' ${ }^{1}$ E. Karaslavova ${ }^{2}$, B. Yaneva ${ }^{1}$ \\ ${ }^{1}$ Department of Periodontology and Oral Diseases, Faculty of Dental Medicine, Medical University - Plovdiv, Bulgaria \\ ${ }^{2}$ Biomedical Analysis - Plovdiv, Bulgaria
}

\begin{abstract}
Aim: To compare the level of diagnostic coincidence between classical (standard) method and VELscope and ViziLite Plus systems in the diagnosis of different oral lesions. Material and methods: 184 oral lesions were examined using classical method, VELscope and ViziLite Plus systems, and after that underwent a pathohistological examination for diagnosis proof. The percentage of diagnostic coincidence for various types of lesions was analyzed for the three methods compared. Results: The results demonstrated the highest coincidence rate for lesions diagnosed with VELscope - 35 (83.3\%), followed by those with classical method - 80 (80.8\%), and those with the application of ViziLite -33 (76.7\%). In premalignant and malignant lesions, the highest percentage of diagnostic coincidence was reported using the classical method - 14 (93.3\%), for non-malignant lesions using VELscope - 28 (84.8\%), for inflammatory and reactive lesions using VELscope - 14 (82.4\%) and for lesions associated with general disease and systemic medication again using VELscope - 11 (91.7\%). Conclusion: Non-invasive methods, tested in the study, have different diagnostic properties when differentiating particular clinical types of lesions. They are highly sensitive to changes in the oral mucosa but the final diagnosis must always be proved with biopsy.
\end{abstract}

Key words: oral lesions, diagnostics, VELscope, ViziLite, premalignant and malignant oral lesions

Corresponding author: Nikolay Nikolov, Blvd. Hristo Botev 3, Bg-4000 Plovdiv, e-mail: dr_n.nikolov@abv.bg

REVISED: 22 July 2020; ACCEPTED :23 June 2020

\section{INTRODUCTION}

A lot of new non-invasive diagnostic methods are created to support traditional oral screening for optimizing early detection of oral precancerous and neoplastic lesions. One of these oral tissue imaging systems is available under the name ViziLite ${ }^{\circledR}$ Plus (Zila Pharmaceuticals, Inc., Phoenix, AZ). The system includes a hemiluminescent light source that illuminates tissues with blue light and is used to detect abnormal changes in the oral mucosa. The ViziLite ${ }^{\circledR}$ Plus system is based on the reflection of light from hyperkeratotic areas of the oral mucosa. They appear white in contrast to the unchanged mucosa that absorbs light and appears darker [1]. Epstein et al. (2006) and Kerr et al. (2006) demonstrated that ViziLite ${ }^{\circledR}$ can potentially assist in the detection of oral premalignant lesions by improving the brightness and clarity of the observed oral structures [4, 9]. By adding bright blue light, the keratinized areas are localized more effectively $[4,14,18]$. Some studies report that ViziLite ${ }^{\circledR}$ does not assist significantly in the identification of oral lesions $[5,20]$. 
Another non-invasive diagnostic option is the additional tissue autofluorescence, that recently has been proven as promising adjuvant diagnostic tool $[6,7$, $11,13]$. Each of our cells contains molecules capable for autofluorescence, especially when activated (excited) by specific light waves [2, 13, 21, 25, 26]. VELscope $^{\circledR}$ (LED Dental, Inc., White Rock, BC, Canada) is a technical device used to visualize tissue autofluorescence in the oral cavity. VELscope ${ }^{\circledR}$ uses blue light with a wavelength of about $436 \mathrm{~nm}$. This wavelength stimulates the green fluorescence of healthy tissues until the altered tissues do not fluoresce and remain dark [3]. For example oral carcinoma and precarcinoma demonstrate loss of autofluorescence, which is mainly due to the reduction of fluorescing structures associated with collagen in the stroma underlying the neoplastic lesion [24].

\section{PURPOSE}

The aim of the present study was to evaluate the diagnostic coincidence between classical (standard) method and the systems VELscope ${ }^{\circledR}$ and ViziLite ${ }^{\circledR}$ Plus in diagnostics of particular clinical types of oral lesions. Informed consent was obtained from each of the participant involved in this study. The study was conducted in accordance with the 1964 Declaration of Helsinki and its subsequent amendments.

\section{MATERIALS AND METHODS}

232 oral lesions of patients (aged $50.26 \pm 16.07,60$ male and 172 female) from the Department of Oral Pathology, Faculty of Dental Medicine, Medical University Plovdiv were examined using the three methods - classical, VELscope and ViziLite, in the period from 01 January, 2013 till 31 July, 2016. 184 of them were subject of the following study and have undergone a pathohistological examination. Histological materials were observed in the Department of Pathological Anatomy, Faculty of Medicine, Medical University of Plovdiv and Medical Diagnostic Laboratory, SMDL Trimed, Plovdiv. The percentage of diagnostic coincidence for the different types of lesions

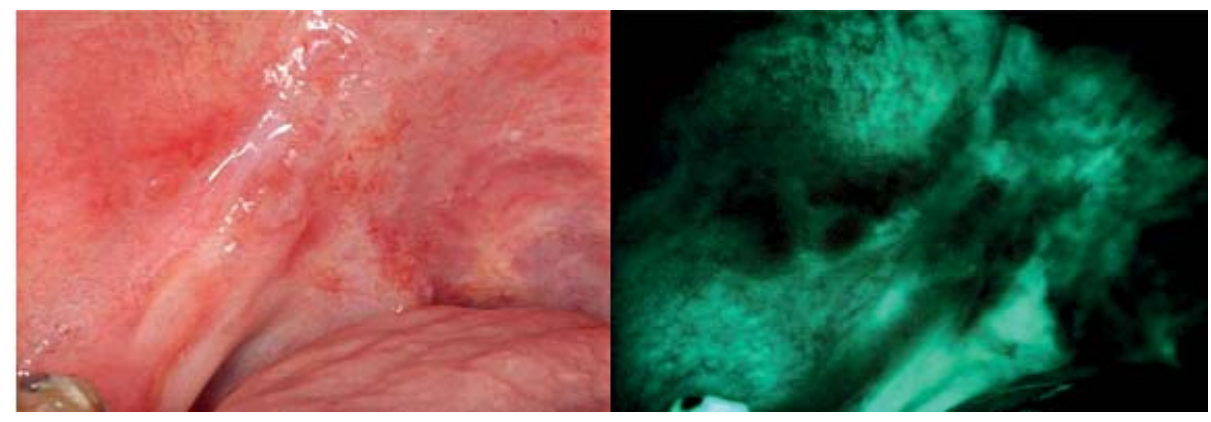

Fig. 2. Areas of reduced fluorescence (dark areas) are considered to be suspected for epidermal dysplasia or even squamous cell carcinoma (positive finding), whereas normal healthy mucosa appears bright green
5 clinical groups - inflammatory and reactive lesions, infectious lesions, lesions related to common diseases and medication administration, pigment lesions, and premalignant and malignant lesions.

Thorough examination of the oral mucosa and classification of the lesions according to the guidelines of the WHO for epidemiological diagnosis of oral lesions was performed. Lesion characteristics, localization, spread, etiological or related factors, dental status, periodontal status, trauma and others were analyzed.

Methods used for early non-invasive diagnosis of oral (premalignant) lesions in the oral cavity using light were:

\section{Autofluorescence}

The VELscope system uses blue light with a peak intensity of $436 \mathrm{~nm}$. This particular wavelength stimulates green fluorescence of healthy tissues, while the altered tissues are non-fluorescent and remain dark (Fig. 1 and 2).

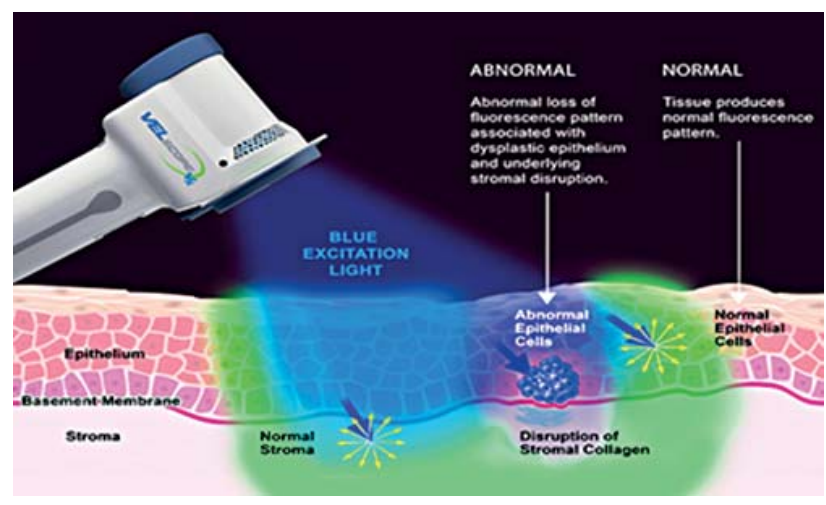

Fig. 1. Diagram showing the principle of VELscope diagnosis based on mucosal biofluorescence in the oral cavity (source: www. velscope.com)

\section{Chemiluminescence}

The ViziLite Plus system is based on the reflection of light from hyperkeratotic areas of the oral mucosa, thereby they appear white in contrast to the unchanged mucosa, which absorbs light and appears dark (Fig. 3). was analyzed in the three compared methods, with the data being considered for: the entire sample without detailed differentiation of particular oral lesions; in the differentiation of lesions into two groups - premalignant and other lesions; in structuring the lesions in 


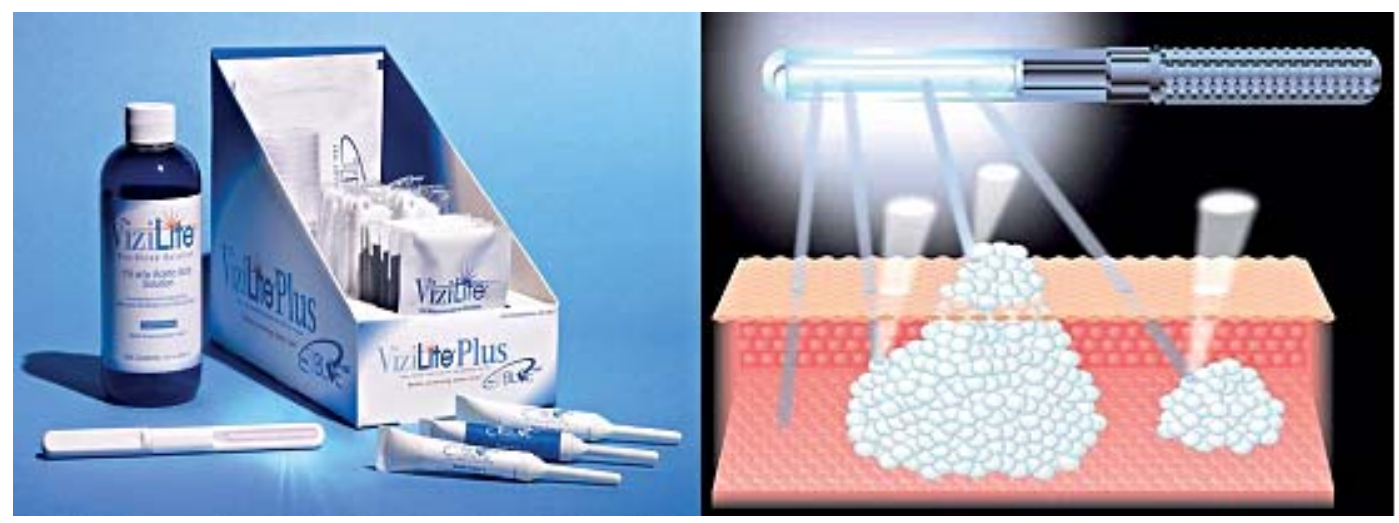

incidence was found in pigment lesions - 3 (42.9\%). It was demonstrated that the difference between the relative proportions of the presence of diagnostic coincidence in

Fig. 3. A. ViziLite Plus system kit. B. Diagram demonstrating the principle of ViziLite Plus system. (source: www.vizilite.com )

\section{Statistical analysis}

The results were analyzed using SPSS Ver. 19 and were considered statistically significant at the level $\alpha=0.05$. Descriptive analysis was used to describe the structure of processes and occurrences and $\chi^{2}$ analysis (Chi-squared test) - to establish relations between qualitative variables.

\section{RESULTS}

Structural distribution of oral lesions (definitive diagnoses are set up according to pathohistological results) depending on whether there was a coincidence with the initial diagnosis is presented in Table 1. A coincidence of diagnoses was reported in a total of 148 $(80.4 \%)$ of the observed oral lesions. The highest correlation between pathohistological and preliminary diagnosis was observed in lesions related to common diseases and medication use - 59 (88.1\%) and in premalignant and malignant lesions - 27 (87.1\%). The observed coincidence in inflammatory and reactive lesions was 53 (77.9\%). The lowest reported co- the different groups of oral lesions was statistically significant - Likelihood Ratio $=14,211, p=0.007$.

When comparing diagnostic methods it was observed whether there was a significant difference in the coincidence of preliminary diagnoses of oral lesions with the final ones, whereas classical and new non-invasive techniques - VELscope and ViziLite were compared (Table 2). In total for all lesions the highest coincidence rate was found for lesions diagnosed with VELscope - 35 (83.3\%), followed by those with the classical method - $80(80.8 \%)$ and lastly confirmed diagnoses using ViziLite - 33 (76.7\%).

The difference in the relative proportions of coincidence rate of diagnoses between the compared methods was not statistically significant (Likelihood Ratio $=0.598 ; p=0.741)$.

In $27(87.1 \%)$ of the premalignant and malignant lesions there was a diagnostic coincidence (Table 3 ). In the other lesions the coincidence in diagnoses was in $121(79.1 \%)$ of the cases. The difference of $8 \%$ in diagnostic coincidence observed between premalignant and other lesions was not statistically significant - Pearson Chi-Square $=1,051, p=0.305$.

Table 1. Diagnostic coincidence in particular clinical groups of oral lesions

\begin{tabular}{|c|c|c|c|c|c|c|c|}
\hline & & & \multicolumn{2}{|c|}{ Coincidence } & \multirow{2}{*}{ TOTAL } & \multirow{2}{*}{$\begin{array}{l}\text { Likelihood } \\
\text { Ratio }\end{array}$} & \multirow{2}{*}{$p$} \\
\hline & & & NO & YES & & & \\
\hline \multirow{10}{*}{$\begin{array}{l}\text { Clinical groups oral le- } \\
\text { sions }\end{array}$} & \multirow{2}{*}{$\begin{array}{c}\text { Inflammatory and reactive } \\
\text { lesions }\end{array}$} & $\mathrm{N}$ & 15 & 53 & 68 & 14,211 & 0,007 \\
\hline & & $\%$ & $22,1 \%$ & $77,9 \%$ & $100,0 \%$ & & \\
\hline & \multirow{2}{*}{ Infectious lesions } & $\mathrm{N}$ & 5 & 6 & 11 & & \\
\hline & & $\%$ & $45,5 \%$ & $54,5 \%$ & $100,0 \%$ & & \\
\hline & \multirow{2}{*}{$\begin{array}{l}\text { Related to common diseases } \\
\text { (incl. autoimmune diseases) } \\
\text { and medication intake }\end{array}$} & $\mathrm{N}$ & 8 & 59 & 67 & & \\
\hline & & $\%$ & $11,9 \%$ & $88,1 \%$ & $100,0 \%$ & & \\
\hline & \multirow{2}{*}{ Pigmented lesions } & $\mathrm{N}$ & 4 & 3 & 7 & & \\
\hline & & $\%$ & $57,1 \%$ & $42,9 \%$ & $100,0 \%$ & & \\
\hline & \multirow{2}{*}{$\begin{array}{l}\text { Premalignant and malignant } \\
\text { lesions }\end{array}$} & $\mathrm{N}$ & 4 & 27 & 31 & & \\
\hline & & $\%$ & $12,9 \%$ & $87,1 \%$ & $100,0 \%$ & & \\
\hline \multirow[t]{2}{*}{ Total } & & $\mathrm{N}$ & 36 & 148 & 184 & & \\
\hline & $\%$ & $19,6 \%$ & $80,4 \%$ & $100,0 \%$ & & & \\
\hline
\end{tabular}


Table 4 demonstrates the distribution of coincidences in diagnoses in premalignant and malignant lesions and all other lesions according to the three methods used for diagnosis of oral lesions. In the premalignant and malignant lesions the highest percentage of diagnostic coincidence was reported using the classical method - 14 (93.3\%), whereas in ViziLite it was $6(85.7 \%)$ and the lowest was found in VELscope group - 7 (77.8\%). For non-malignant lesions the tendency was the opposite - the lowest percentage of diagnostic coincidence was observed in ViziLite group $-27(75.0 \%)$, followed by the classical method - 66 (78.6\%), and diagnosing with VELscope dem- onstrates the highest $-28(84.8 \%)$. No statistically significant difference in the compared groups was detected $-p>0.05$, which may be due to the limited number of patients or to second-order errors made.

Inflammatory and reactive lesions demonstrated the highest coincidence rate in the VELscope group - 14 $(82.4 \%)$ and the lowest confirmed diagnosis in the ViziLite group - $5(62,5 \%)$ (Table 5). Confirmation of diagnoses in lesions related to general disease and medication was more likely to be achieved when diagnosed with VELscope - $11(91.7 \%)$ than when diagnosed with ViziLite $-19(82,6 \%)$.

Table 2. Diagnostic coincidences of the three diagnostic methods compared.

\begin{tabular}{|c|c|c|c|c|c|c|c|}
\hline & & & \multicolumn{2}{|c|}{ Coincidence } & \multirow{2}{*}{ Total } & \multirow{2}{*}{$\begin{array}{l}\text { Likelihood } \\
\text { Ratio }\end{array}$} & \multirow[b]{2}{*}{$p$} \\
\hline & & & NO & YES & & & \\
\hline \multirow{6}{*}{ Method used } & \multirow{2}{*}{ Classical method } & $\mathrm{N}$ & 19 & 80 & 99 & 0,598 & 0,741 \\
\hline & & $\%$ & $19,2 \%$ & $80,8 \%$ & $100,0 \%$ & & \\
\hline & \multirow{2}{*}{ VELscope } & $\mathrm{N}$ & 7 & 35 & 42 & & \\
\hline & & $\%$ & $16,7 \%$ & $83,3 \%$ & $100,0 \%$ & & \\
\hline & \multirow{2}{*}{ ViziLite } & $\mathrm{N}$ & 10 & 33 & 43 & & \\
\hline & & $\%$ & $23,3 \%$ & $76,7 \%$ & $100,0 \%$ & & \\
\hline \multirow[t]{2}{*}{ Total } & & $\mathrm{N}$ & 36 & 148 & 184 & & \\
\hline & $\%$ & $19,6 \%$ & $80,4 \%$ & $100,0 \%$ & & & \\
\hline
\end{tabular}

Table 3. Differences in diagnostic coincidences between premalignant and malignant lesions and other lesions

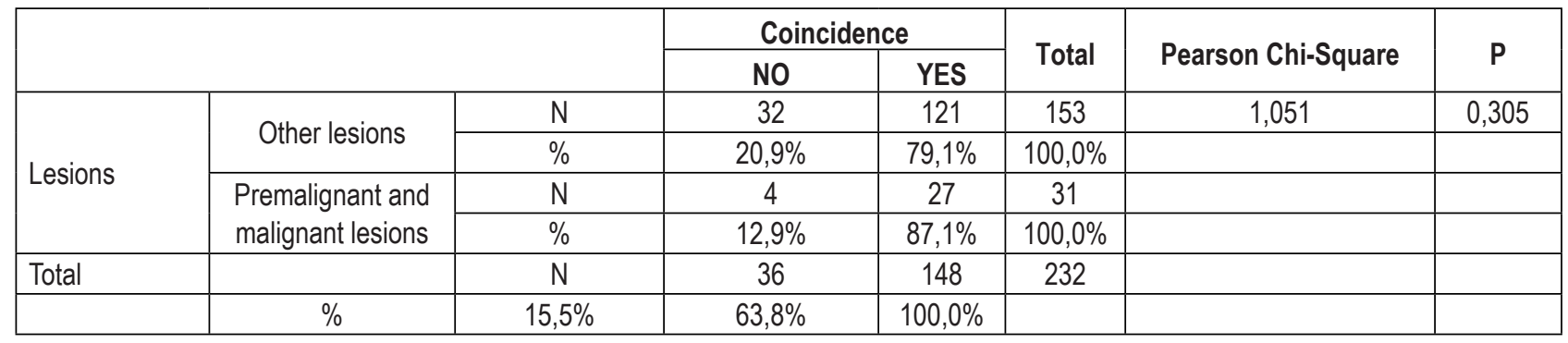

Table 4. Differences in diagnostic coincidences between premalignant and malignant lesions and other lesions depending on the diagnostic method

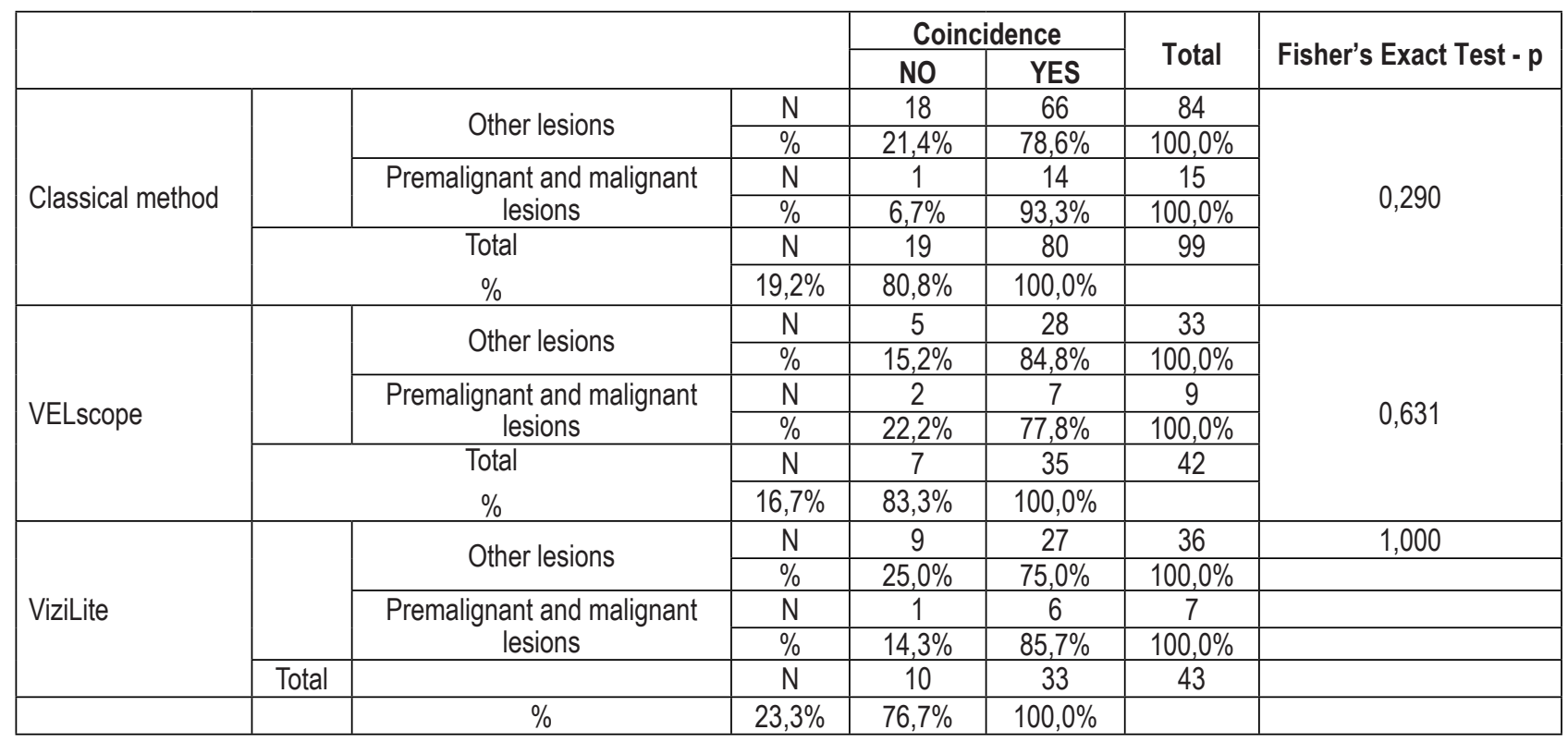


Table 5. Diagnostic coincidences in different clinical groups of lesions depending on the diagnostic method

\begin{tabular}{|c|c|c|c|c|c|c|c|c|}
\hline & & & & Coil & dence & & Likelihood & \\
\hline & & & & NO & Yes & Total & Ratio & $p$ \\
\hline & & Inflammatory and reactive & $\mathrm{N}$ & 9 & 34 & 43 & & \\
\hline & & lesions & $\%$ & $20,9 \%$ & $79,1 \%$ & $100,0 \%$ & & \\
\hline & & Infectious lesinns & $\mathrm{N}$ & 3 & 2 & 5 & & \\
\hline & & 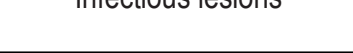 & $\%$ & $60,0 \%$ & $40,0 \%$ & $100,0 \%$ & & \\
\hline & & Lesions related to common & $\mathrm{N}$ & 3 & 29 & 32 & & \\
\hline $\begin{array}{l}\text { Classical } \\
\text { method }\end{array}$ & $\begin{array}{l}\text { Clinical groups } \\
\text { oral lesions }\end{array}$ & $\begin{array}{l}\text { diseases (incl. autoimmune } \\
\text { diseases) and medication } \\
\text { intake }\end{array}$ & $\%$ & $9,4 \%$ & $90,6 \%$ & $100,0 \%$ & 14,211 & 0,007 \\
\hline & & Dirmontod locinne & $\mathrm{N}$ & 3 & 1 & 4 & & \\
\hline & & Pigmented Iesions & $\%$ & $75,0 \%$ & $25,0 \%$ & $100,0 \%$ & & \\
\hline & & Premalignant and malignant & $\mathrm{N}$ & 1 & 14 & 15 & & \\
\hline & & lesions & $\%$ & $6,7 \%$ & $93,3 \%$ & $100,0 \%$ & & \\
\hline & & Total & $\mathrm{N}$ & 19 & 80 & 99 & & \\
\hline & & $\%$ & $19,2 \%$ & $80,8 \%$ & $100,0 \%$ & & & \\
\hline & & Inflammatory and reactive & $\mathrm{N}$ & 3 & 14 & 17 & & \\
\hline & & lesions & $\%$ & $17,6 \%$ & $82,4 \%$ & $100,0 \%$ & & \\
\hline & & Infectious lecions & $\mathrm{N}$ & 0 & 1 & 1 & & \\
\hline & & 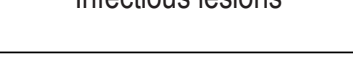 & $\%$ & $0,0 \%$ & $100,0 \%$ & $100,0 \%$ & & \\
\hline & & Lesions related to common & $\mathrm{N}$ & 1 & 11 & 12 & & \\
\hline VELscope & $\begin{array}{l}\text { Clinical groups } \\
\text { oral lesions }\end{array}$ & $\begin{array}{l}\text { diseases (incl. autoimmune } \\
\text { diseases) and medication } \\
\text { intake }\end{array}$ & $\%$ & $8,3 \%$ & $91,7 \%$ & $100,0 \%$ & 1,765 & 0,779 \\
\hline & & Piamented l lecinne & $\mathrm{N}$ & 1 & 2 & 3 & & \\
\hline & & rigmented resions & $\%$ & $33,3 \%$ & $66,7 \%$ & $100,0 \%$ & & \\
\hline & & Premalignant and malignant & $\mathrm{N}$ & 2 & 7 & 9 & & \\
\hline & & lesions & $\%$ & $22,2 \%$ & $77,8 \%$ & $100,0 \%$ & & \\
\hline & & Total & $\mathrm{N}$ & 7 & 35 & 42 & & \\
\hline & & $\%$ & $16,7 \%$ & $83,3 \%$ & $100,0 \%$ & & & \\
\hline & & Inflammatory and reactive & $\mathrm{N}$ & 3 & 5 & 8 & & \\
\hline & & lesions & $\%$ & $37,5 \%$ & $62,5 \%$ & $100,0 \%$ & & \\
\hline & & Infectious lecions & $\mathrm{N}$ & 2 & 3 & 5 & & \\
\hline & & 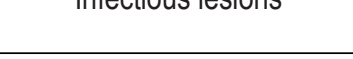 & $\%$ & $40,0 \%$ & $60,0 \%$ & $100,0 \%$ & & \\
\hline & Clinical groups & Lesions related to common & $\mathrm{N}$ & 4 & 19 & 23 & & \\
\hline Vizilite & Oral lestoris & $\begin{array}{l}\text { diseases (incl. autoimmune } \\
\text { diseases) and medication } \\
\text { intake }\end{array}$ & $\%$ & $17,4 \%$ & $82,6 \%$ & $100,0 \%$ & 2,332 & 0,507 \\
\hline & & Premalignant and malignant & $\mathrm{N}$ & 1 & 6 & 7 & & \\
\hline & & lesions & $\%$ & $14,3 \%$ & $85,7 \%$ & $100,0 \%$ & & \\
\hline & Total & & $\mathrm{N}$ & 10 & 33 & 43 & & \\
\hline & & $\%$ & $23,3 \%$ & $76,7 \%$ & $100,0 \%$ & & & \\
\hline
\end{tabular}




\section{DISCUSSION}

The overall percentage of diagnostic coincidences (including all oral lesions) was highest with the use of VELscope - $35(83.3 \%)$ and lowest with ViziLite - $33(76.6 \%)$, which is consistent with findings of other studies examining diagnostic capabilities of the ViziLite method in oral pathology $[5,15]$. On the other hand, the result of the diagnostic efficiency of the new methods, in particular of ViziLite, is in contrast to the data obtained by other authors $[4,9,20]$ (Table 2).

In total for all lesions, when they are divided into 2 main subgroups: group 1 - premalignant and malignant lesions and group 2 - all other lesions, an $8 \%$ higher coincidence rate is observed for pre-malignant and malignant lesions in comparison to all other lesions (group 2) (Table 3). Thus, the result obtained when comparing the diagnostic effectiveness of the three methods is difficult to explain. The highest percentage of diagnostic coincidence is reported for premalignant and malignant lesions when diagnosed by the classical method - 14 (93.3\%) (Table 4). According to our results the diagnostic coincidence in the group of lesions diagnosed with VELscope has the lowest percentage - 7 (77.8\%), and for the lesions diagnosed with ViziLite it is 6 $(85,7 \%)$. The findings of this study do not support the findings of Epstein et al. (2006), Kerr et al. (2006), and other authors who conclude that ViziLite and VELscope can potentially help more effectively than the classical method in the detection of oral premalignant lesions $[4,8,9,12,22,23]$. A lot of studies report the lack of ability for those new methods to identify premalignant and malignant lesions $[15,16]$. The present findings are consistent with other studies reporting that ViziLite does not significantly help in the accurate identification of oral lesions [5, 20]. A possible explanation of our results is that the recent study includes very limited number of premalignant and malignant lesions diagnosed with VELscope and ViziLite. In the group of premalignant lesions, when comparing the number rather than the relative proportions, one or two of these lesions in all three methods show that the final diagnosis does not coincide with the initial one. The rest of the lesions other than premalignant and malignant ones are most accurately diagnosed with VELscope $-84.8 \%$, followed by the classic oral examination $-78.6 \%$, and last with ViziLite $-75.0 \%$ (Table 4).

According to the present results highest coincidence rate is observed when diagnosing inflammatory and reactive lesions with VELscope - 14 (82.4\%) and the lowest diagnostic coincidence rate for this group of lesions is achieved when using ViziLite $-5(62,5 \%)$.
The lower diagnostic efficacy of ViziLite especially for inflammatory and reactive lesions is also demonstrated by Farah C.S. and McCullough M.J. in 2007 and Ram S. and Siar C.H. in 2005 [5, 20]. The difference in percentages found between both diagnostic modalities is nearly $20 \%$ in favor to VELscope, and according to current data, VELscope seems to be a more reliable method of diagnosing inflammatory and reactive lesions than ViziLite.

Furthermore VELscope demonstrates better diagnostic abilities in diagnosing lesions related to general disease and medication intake - $11(91.7 \%)$ in comparison to ViziLite - 19 (82.6\%). The percentage of diagnostic coincidences for this group of lesions when using the classical method is comparable to the result obtained with the VELscope examination.

It should be noted that most of the literature data on the topic relates to: more effective detection of occult oral lesions; more accurate localization the edges of the oral lesion for complete excision; determination of the most appropriate biopsy area; rather than exact diagnosis $[10,12,17,19]$.

\section{CONCLUSION}

Several scientific and practical highlights of the present study are outlined. When observing all oral lesions, the best diagnostic efficiency was achieved with the application of VELscope, followed by the classical method and lastly with ViziLite. There is a significantly greater diagnostic coincidence in premalignant and malignant lesions than in all other oral lesions, demonstrating high but specifically targeted and highly risk-oriented professionalism of dental practitioners. It should be noted that in premalignant and malignant lesions the highest diagnostic efficiency was achieved when using the classical method, followed by ViziLite and finally by VELscope. For all other lesions, the diagnostic coincidences are highest in VELscope, followed by the classical method and ViziLite. Our data demonstrate that in the two largest groups of oral lesions: the first - related to general disease and medication and the second - inflammatory and reactive diseases, VELscope presents significantly better diagnostic efficiency than ViziLite.

In summary, the above-mentioned instrumental methods have a certain diagnostic specificity, standing out both their advantages and highlighting points of discussion that require practical refinement of the diagnostic approach in individual oral lesions.

Disclosure summary: The authors have nothing to disclose. 


\section{REFERENCES}

1. Nikolov N. Съвременни неинвазивни методи за оценка на оралните преканцерози. Dental Tribune Bulgarian Edition. 2006.

2. Betz CS, Mehlmann M, Rick K, Stepp H, et al. Autofluorescence imaging and spectroscopy of normal and malignant mucosa in patients with head and neck cancer. Lasers Surg Med 1999; 25: 323-334.

3. Bouquot JE, Gorlin RJ. Leukoplakia, lichen planus and other oral keratoses in 23,616 white Americans over the age of 35 years. Oral Surg Oral Med Oral Pathol 1986; 61:373-381.

4. Epstein JB, Gorsky M, Lonky $S$ et al. The efficacy of oral lumenoscopy (ViziLite) in visualizing oral mucosal lesions. Spec Care Dentist 2006;26:171-74.

5. Farah CS, McCullough MJ. A pilot case control study on the efficacy of acetic acid wash and chemiluminescent illumination (ViziLite trade mark) in the visualisation of oral mucosal white lesions. Oral Oncol 2007;43:820-24.

6. Huh WK, Cestero RM, Garcia FA et al. Optical detection of high-grade cervical intraepithelial neoplasia in vivo: results of a 604-patient study. Am J Obstet Gynecol 2004;190:1249-57.

7. Kara MA, Peters FP, Fockens $P$ et al. Endoscopic video-autofluorescence imaging followed by narrow band imaging for detecting early neoplasia in Barrett's esophagus. Gastrointest Endosc 2006;64:176-85.

8. Kelloff GJ, Sullivan DC, Baker H et al. Workshop on imaging science development for cancer prevention and preemption. Cancer Biomark, 2007 3, 1-33

9. Kerr AR, Sirois DA, Epstein JB. Clinical evaluation of chemiluminescent lighting: an adjunct for oral mucosal examinations. J Clin Dent 2006; 17:59-63.

10. Kois JC, Truelove E. Detecting oral cancer: A new technique and case reports. Dent. Today 2006;25(10), 94, 96-97

11. Lam S, Kennedy $\mathrm{T}$, Unger M et al. Localization of bronchial intraepithelial neoplastic lesions by fluorescence bronchoscopy. Chest 1998;113:696-702.

12. Laronde DM, Poh CF, Williams PM et al. A magic wand for the community dental office? Observations from the British Columbia oral cancer prevention program. J. Can. Dent. Assoc., 2007; 73(7), 607-609

13. Matsuda T, Saito $\mathrm{Y}, \mathrm{Fu} \mathrm{KI}$ et al. Does autofluorescence imaging videoendoscopy system improve the colonoscopic polyp detection rate? A pilot study. Am J Gastroenterol 2008;103:1926-32.

14. McNamara, K. (2009). The Role of Direct Visual Fluorescent Examination (VELscope) in Tumor Margin Delineation and Routine Screening of the Oral Cavity. (Electronic Thesis or Dissertation). Retrieved from https://etd.ohiolink.edu/

15. Oh ES, Laskin DM. Efficacy of the ViziLite system in the identification of oral lesions. J. Oral Maxillofac. Surg., 2007; 65(3), 424-426

16. Patton LL, Epstein JB, Kerr AR. Adjunctive techniques for oral cancer examination and lesion diagnosis. J. Am. Dent. Assoc., 2008;139, 896-905

17. Paulis $M$. The influence of patient education by the dental hygienist: acceptance of the fluorescence oral cancer exam. J. Dent. Hygiene, 2009; 83(3), 134-140

18. Pierre M, Lane PM, Whitehead TGP, Zeng H, et al. Simple device for the direct visualization of oral-cavity. J Biomed Optics 2006; 11:024-26.

19. Poh CF, Ng SP, Williams PM et al. Direct fluorescence visualization of clinically occult high-risk oral premalignant disease using a simple hand-held device. Head Neck, 2007; 29(1), 71-76

20. Ram S, Siar $\mathrm{CH}$. Chemiluminescence as a diagnostic aid in the detection of oral cancer and potentially malignant epithelial lesions. Int J Oral Maxillofac Surg 2005;34:521-527.

21. Roblyer D, Kurachi C, Stepanek V et al. Objective detection and delineation of oral neoplasia using autofluorescence imaging. Cancer Prev Res (Phila Pa) 2009; 2:423-431.

22. Rosin MP, Poh CF, Elwood JM et al. New hope for an oral cancer solution: together we can make a difference. J. Can. Dent. Assoc., 2008; 74(3), 261-266.

23. Schwimmer, E. Die idiopathischen Schleimhautplauques der Mundhohle (Leukoplakia buccalis). Arch Dermat Syph 9:611570, 1877.

24. Shin D, Vigneswaran N, Gillenwater A, Richards-Kortum R. Advances in fluorescence imaging techniques to detect oral cancer and its precursors.Future Oncol. $2010 \mathrm{Jul} ; 6(7): 1143-54$.

25. Skala MC, Riching KM, Gendron-Fitzpatrick $A$ et al. In vivo multiphoton microscopy of NADH and FAD redox states, fluorescence lifetimes, and cellular morphology in precancerous epithelia. PNAS 2007; 104:19494-19499

26. Svistun E, Alizadeh-Naderi R, El-Naggar A et alR. Vision enhancement system for detection of oral cavity neoplasia based on autofluorescence. Head Neck 2004; 26:205-215. 